Research Article

\title{
Electrophysical Properties of Epoxy Composite Materials Filled with Carbon Black Nanopowder
}

\author{
Andriy Buketov (D), ${ }^{1}$ Serhii Smetankin $\mathbb{D D}^{1}{ }^{1}$ Eduard Lysenkov $\mathbb{D}^{\mathrm{D}},{ }^{2}$ Kyrylo Yurenin $\left(\mathrm{D},{ }^{1}\right.$ \\ Oleksandr Akimov $\left(\mathbb{D},{ }^{1}\right.$ Serhii Yakushchenko $₫$, ${ }^{1}$ and Iryna Lysenkova $\mathbb{1}^{2}$ \\ ${ }^{1}$ Department of Transport Technologies, Kherson State Maritime Academy, 20 Ushakova Ave., Kherson 73000, Ukraine \\ ${ }^{2}$ Department of Intellectual Information Systems, Petro Mohyla Black Sea National University, 1068 Desantnykiv Str., \\ Mykolaiv 54003, Ukraine
}

Correspondence should be addressed to Serhii Yakushchenko; yakushchenko.sv@gmail.com

Received 4 May 2020; Revised 22 June 2020; Accepted 29 June 2020; Published 21 July 2020

Academic Editor: Claude Estournès

Copyright (c) 2020 Andriy Buketov et al. This is an open access article distributed under the Creative Commons Attribution License, which permits unrestricted use, distribution, and reproduction in any medium, provided the original work is properly cited.

\begin{abstract}
The effect of carbon black (CB) nanopowder on the electrical properties of polymer composite systems based on the epoxy resin is investigated using the method of impedance spectroscopy. It is established that the electrical and dielectric properties of the studied systems significantly depend on the nanofiller content. It is found that electrical conductivity and dielectric constant exhibit percolation behavior when the filler's content increases. In this case, the electrical conductivity increases exponentially, indicating the formation of filler electrically conductive mesh inside the polymer matrix. A small jump in electrical conductivity when reaching the percolation threshold indicates the formation of indirect contacts between the particles. The value of the percolation threshold of conductivity is $8 \%$. It is shown that the dielectric constant of epoxy nanosystems is almost unchanged in the frequency range of $10^{2}-10^{5} \mathrm{~Hz}$. It is related to the structural features of the filler particles, which ensure the existence of a minimal dielectric gradient between the matrix and the filler. It is found that the dielectric constant of the studied systems also shows percolation behavior. The obtained material based on the epoxy matrix is characterized by a high value of dielectric constant, which at a carbon black nanopowder content of $29 \%$ is 4680 . This material is characterized by relative frequency invariance and a high value of dielectric constant, so it has great potential for practical application.
\end{abstract}

\section{Introduction}

Polymer composite materials (PCMs) have been the subject of intense research during the last three decades. Particular attention is paid to the electrophysical properties of such materials. Traditionally, polymers are considered to be electrically insulating materials due to the low concentration of free charge carriers in their composition. However, the electrical characteristics of the polymers can be improved by including the electrically conductive filler in their composition. The electrically conductive polymer composites thus obtained combine the good mechanical properties, flexibility, and ease of processing of polymeric materials with the unique electrical properties of conductive particles [1].

Polymer composites containing electrically conductive filler have great potential for application in various fields, for example, for the creation of various sensors, antistatic shielding, and the protection against electromagnetic radiation, as well as it can be used in electronics and aerospace industry $[2,3]$. Carbon fillers, such as carbon nanotubes, fullerenes, graphene, carbon fibres, and activated carbon nanopowders, are often used for the fabrication of PCM with improved physical-mechanical [4-6] and electrically conductive properties [7-9]. Some authors note the prospect of using carbon black (CB) particles as filler for such materials [10-12]. For example, Mailabari investigated the electrical properties of the system, based on epoxy polymer and carbon black. It is found that the percolation threshold, i.e., the concentration of filler at which there is a sharp jump in electrical conductivity, in such systems is $20-25$ wt. \%. It is shown that the particle size, their content, and morphology influence the electrical resistance of the composite [11]. 
However, Mamunya et al., studying a wide range of thermoplastic polymers filled with $\mathrm{CB}$, found that the percolation threshold in such systems varies around 7-9\% and directly depends on the interaction between the polymer matrix and the filler [12]. One way to improve the electrical properties of the system and reduce the percolation threshold is to use nanoscale fillers. Thus, Zhang used nanodispersed $\mathrm{CB}$ to create nanocomposite materials based on the epoxy resin for the manufacture of bending sensors [13]. The mixture of epoxy, hardener, and nano-CB is put into the sample capsule with three stainless steel balls $(4 \mathrm{~mm}$ diameter) and exposed to rapid vibration for $10 \mathrm{~h}$, wherein the action of the steel balls under vibration caused a grinding effect on the materials in the mixture. They found that the introduction of nanosized CB led to a sharp increase in the electrical conductivity of the system, but the percolation threshold was quite large, around 19-24\%. However, Choi et al. reported the positive effect of reducing the size of $\mathrm{CB}$ particles. The carbon black/polymer mixture was placed in the twin-screw extruder's hopper and used to perform meltspinning. They found that the percolation threshold in the nylon-nano-CB system was 3\% [14]. Therefore, the effect of the size, dispersion of $\mathrm{CB}$, and the preparation method of composites on the electrical properties of the polymer matrix are ambiguous. This effect needs further investigation of epoxy-nano-CB systems.

Composite systems based on epoxy polymer and CB have a high potential to produce materials with high dielectric constant. Unlike ceramic fillers, which are traditionally used to create high dielectric permittivity materials, the use of $\mathrm{CB}$ as a filler improves the mechanical properties [15] of such materials and extends their application. For example, $\mathrm{Xu}$ reported the creation of promising materials based on epoxy polymer and CB for using high-capacity capacitors [16]. In this case, the studied materials were characterized by a high value of dielectric constant, which at a frequency of $10 \mathrm{kHz}$ was 2300 . However, the obtained composites showed a significant dependence on the frequency, which is a disadvantage in the creation of capacitors.

To ensure frequency invariance and high values of dielectric constant, it is necessary to use filler particles with a transient structure to create a minimal property gradient between the filler and the polymeric matrix [17]. Particles of nanodispersed CB meet these requirements and have a coreshell structure, where carbon is the nucleus and shells are different functional groups located on the surface of the particle. This filler has a relatively low electrical conductivity, which makes it promising to use CB to create materials with high dielectric constant.

Therefore, the purpose of this work is to study the electrophysical properties of epoxy composite systems filled with nanodispersed $\mathrm{CB}$, to establish the features of the percolation transition and to estimate the prospects of these systems for creation of materials with high dielectric constant.

\section{The Experimental Part}

2.1. Materials. The composites based on epoxy-diane resin ED-20 (Technobudresurs, Kyiv, Ukraine), containing $21.5 \%$ of epoxy groups, are studied. A low-molecular-weight hardener polyethylene polyamine is used for binding the epoxy oligomer (Technobudresurs, Kyiv, Ukraine), which allows hardening the materials at room temperature. The hardener is introduced into the composition in the amount of $10.5 \%$ of the epoxy oligomer.

The carbon black nanopowder (CBN) PowCarbon 2419G (CAS No: 1333-86-4, EINECS No: 215-609-9) (Black Diamond Material Science Co., Ltd., China) is used as a filler. The structural characteristics of nanopowder are specified by the manufacturer. The particle size in the powder, determined by electron microscopy, is $24 \pm 2 \mathrm{~nm}$. The specific surface area of the particles is $145 \pm 20 \mathrm{~m}^{2} / \mathrm{g}$. The filler is introduced in an amount of $0.1-29.00$ wt. \% (hereinafter \%).

2.2. Samples Preparation. The epoxy PCMs are formed using the following technique: heating the resin to a temperature $T=353 \pm 2^{\circ} \mathrm{K}$ and holding at a given temperature for a time $\tau=20 \pm 0.1 \mathrm{~min}$. CB is a hygroscopic material $[18,19]$, and it can absorb moisture depending on the particle size, the presence of impurities, and storage conditions, which leads to the clumping (sticking) of the CB powder and complicates the introduction and dispersion in the melt of the polymer. Additionally, the drying of the powder is carried out in a laboratory muffle furnace heated to a temperature $T=383 \pm 2^{\circ} \mathrm{K}$ and holding it at a given temperature for a time $\tau=60 \pm 0.1 \mathrm{~min}$. Then, the dosage of the filler is carried out, and it is subsequently introduced into the epoxy oligomer ED-20 for a time $\tau=1 \pm 0.1 \mathrm{~min}$ and then ultrasonic treatment of the composition over $\tau=1.5 \pm 0.1 \mathrm{~min}$, cooling the composition to room temperature for $\tau=60 \pm 5 \mathrm{~min}$ and the introduction of the hardener and mixing the composition for $\tau=5 \pm 0.1 \mathrm{~min}$. The validation of the PCM is carried out according to the experimentally established regimes: formation of samples and holding them at a temperature $T=293 \pm 2^{\circ} \mathrm{K}$ for $\tau=12.0 \pm 0.1 \mathrm{~h}$, heating with a rate $v=3^{\circ} \mathrm{K} /$ min up to the cross-linking temperature $T=393 \pm 2^{\circ} \mathrm{K}$, holding the samples at this temperature for $T=\tau=2.0 \pm 0.05 \mathrm{~h}$, and slow cooling to the temperature $T=293 \pm 2^{\circ} \mathrm{K}$. In order to stabilize the structural processes in the matrix, the samples are held at a temperature $T=298 \pm 2^{\circ} \mathrm{K}$ for $T=24 \mathrm{~h}$ in air, followed by experimental tests. This preparation technique is obtained experimentally, and it is successfully used to create polymer composite materials with improved functional characteristics $[4,8,15]$.

2.3. Research Technique. Studies of electrical and dielectric properties are performed using the impedance spectroscopy method based on the $Z$-2000 impedance meter. The sample is placed between the electrodes of the cell, and it measures its real $\left(Z^{\prime}\right)$ and imaginary $\left(Z^{\prime \prime}\right)$ parts of the impedance. According to the obtained dependences of complex impedance, the electrical conductivity at alternating current (AC) is determined $\sigma_{\mathrm{AC}}=\left(d / S Z^{\prime}\right)$, where $S$ is the area of the sample and $d$ is the thickness of the sample. The measurements are performed at room temperature in the frequency range $10^{2}-10^{5} \mathrm{~Hz}$. 
The deviation of the values in the studies of electrical and dielectric characteristics in PCM is 3\% of the nominal.

\section{Results and Discussion}

During the study of the electrophysical properties of polymer composites, special attention is paid to the issues related to the processes of charge transfer and accumulation. Taking into account the latest developments in the field of experimental engineering, impedance (dielectric) spectroscopy is one of the methods of their estimation. This method measures the response of the studied system to the changing external electric field, which reflects its structural features and allows to obtain a number of dielectric parameters, such as complex $\varepsilon^{*}=\varepsilon^{\prime}+i \varepsilon^{\prime \prime}\left(\right.$ real $\left(\varepsilon^{\prime}\right)$ and imaginary $\left.\left(\varepsilon^{\prime \prime}\right)\right)$ dielectric constant and electrical conductivity $\left(\sigma^{\prime}\right)$.

\subsection{Features of Conductivity of Epoxy-Carbon Black Nano-} powder Systems. Figure 1 presents frequency dependences of electrical conductivity at alternating current for systems based on epoxy matrix and CBN. The epoxy matrix and composites based on it with different contents of CBN show almost linear increase in electrical conductivity with increasing frequency of electric field.

Figure 1 shows that the nature of the electric field frequency and the amount of the introduced filler significantly affects the electrophysical properties of the materials. Studies show that, at a frequency of $100 \mathrm{~Hz}$, the conductivity of the epoxy oligomer is $3 \times 10^{-9} \mathrm{~S} / \mathrm{cm}[18]$ and $1 \times 10^{-10} \mathrm{~S} / \mathrm{cm}[20]$. In our study, the conductivity of the epoxy oligomer at a frequency of $100 \mathrm{~Hz}$ is $1.1 \times 10^{-9} \mathrm{~S} / \mathrm{cm}$. It should be noted that, with the introduction of the minimum concentration of CBN $(\varphi=1 \%)$ into the epoxy, there is a slight increase in the conductivity of PCM $\left(\sigma=2.16 \times 10^{-9} \mathrm{~S} / \mathrm{cm}\right)$ relative to the matrix. Further increase in the number of $\mathrm{CBN}$ to $\varphi=6 \%$ also leads to a minimum increase in this parameter, and it is only $\sigma=3.6 \times 10^{-9} \mathrm{~S} / \mathrm{cm}$. A similar trend is observed with the subsequent introduction of $\mathrm{CBN}$ in the amount of $\varphi=17.29 \%$ (Figure 1), where significant changes in the conductivity, more than one or two orders of magnitude at the frequency of $100 \mathrm{~Hz}$, are also not recorded, ranging from $5.8 \times 10^{-9}$ to $4.2 \times 10^{-8} \mathrm{~S} / \mathrm{cm}$. The results of experimental studies at frequencies of 50 and $100 \mathrm{kHz}$ showed a characteristic increase in the conductivity of PCM. It should be emphasized that the conductivity of PCM with the maximum concentration of CBN $(\varphi=29 \%)$ at the minimum $(100 \mathrm{~Hz})$, the average $(1 \mathrm{kHz})$, and the maximum frequencies $(100 \mathrm{kHz})$ shows an increase of only one and a half order of magnitude, which is not completely consistent with results presented in other papers $[14,15]$.

Figure 1 shows that, with increasing frequency, the electrical conductivity for all studied systems increases. At the same time, the graphs do not show a plateau of conductivity, which allows determining the electrical conductivity at direct current $\left(\sigma_{\mathrm{DC}}\right)$. The absence of a DC plateau is probably due to interfacial polarization (Maxwell-Wagner-Sillars (MWS) polarization) that is caused by the accumulation of bulk charge at the interface of the CB

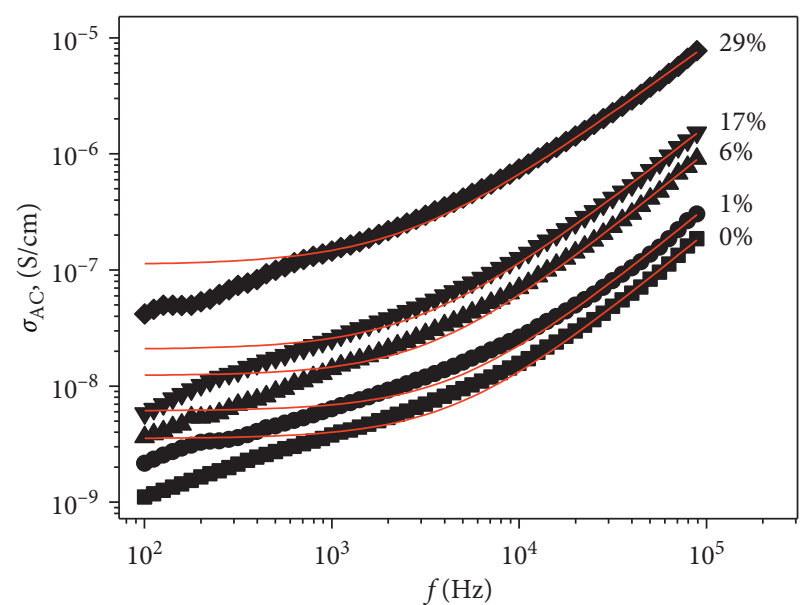

FIgURE 1: Electrical conductivity at alternating current vs. frequency for systems based on epoxy matrix containing CBN. The solid line is the Jonscher model (equation (1)).

polymer, which usually occurs in heterogeneous systems that consist of components with different conductivities [21]. MWS polarization for heterogeneous nanofilled polymer systems is observed in the frequency range from $10^{-3}$ to $10^{3} \mathrm{~Hz}$, which correlates well with the results shown in Figure 1. However, the latent plateau of direct current conductivity in systems with MWS polarization effects can be determined using the Jonscher model, as was done by Povilas et al. for pure epoxy [18].

At low frequencies, charge carriers transfer a large distance inside the composite to change the direction of the electric field. In this case, the average distance transferred by charge carriers at frequencies smaller than the critical frequency is greater than the correlation length (the size of the largest finite size cluster). At high frequencies, the electrical conductivity is realized by hopping the charge carriers between the localized states, so an almost linear increase in the $\sigma(\omega)$ graph is observed [22]. AC conductivity in disordered solids $\sigma(\omega)$ exhibits universal behavior, and it is described by the Jonscher power law [23]:

$$
\sigma(\omega)=\sigma_{\mathrm{DC}}+\sigma_{\mathrm{AC}}=\sigma_{\mathrm{DC}}+A \omega^{n},
$$

where $\sigma_{\mathrm{DC}}$ is the direct current electrical conductivity (frequency-independent behavior), $\omega$ is the angular frequency of the applied electric field $(\omega=2 \pi f), A$ is the preexponential factor, and $n$ is the exponent. The $n$ value represents the degree of interaction between the charge carriers and the surrounding environment.

The obtained frequency dependences of the electrical conductivity of the epoxy nanosystem (Figure 1) are modelled within equation (1). The calculated parameters of equation 1 are given in Table 1.

Table 1 and Figure 2 show that the parameter of interaction $n$ decreases linearly with increasing content of filler in the system. This indicates a decrease in the degree of interaction between the charge carriers and the matrix, which is a consequence of the considerable aggregation of CBN particles into denser aggregates, in which the interaction area with the epoxy polymer is much smaller. The 
TAble 1: Parameters of Jonscher's model (equation (1)).

\begin{tabular}{lccc}
\hline CBN content $(\%)$ & $\sigma_{\text {DC }}(\mathrm{S} / \mathrm{cm})$ & $A(\mathrm{~S} / \mathrm{cm})$ & $n$ \\
\hline 0 & $3.5 \cdot 10^{-9}$ & $4.9 \cdot 10^{-14}$ & 1.33 \\
1 & $6.1 \cdot 10^{-9}$ & $8.5 \cdot 10^{-14}$ & 1.32 \\
6 & $1.2 \cdot 10^{-8}$ & $2.4 \cdot 10^{-13}$ & 1.32 \\
17 & $2.0 \cdot 10^{-8}$ & $8.5 \cdot 10^{-13}$ & 1.26 \\
29 & $1.1 \cdot 10^{-7}$ & $1.0 \cdot 10^{-11}$ & 1.18 \\
\hline
\end{tabular}

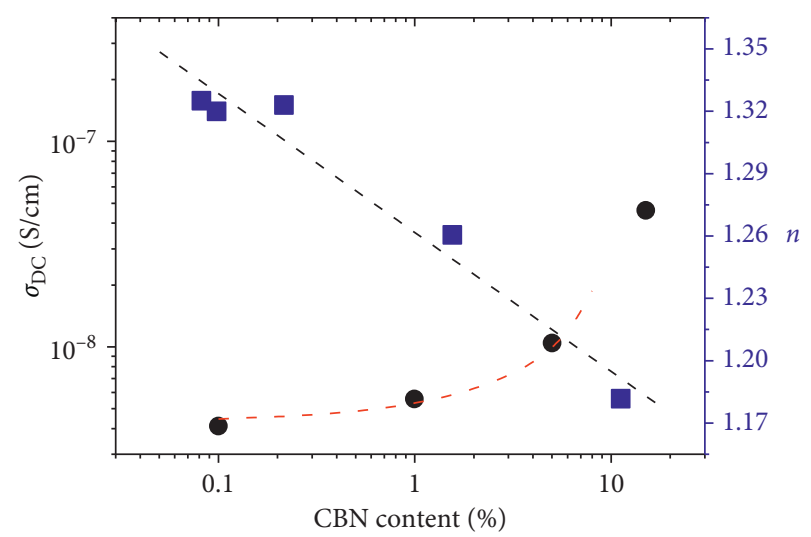

FIGURE 2: Direct current conductivity and interaction parameter vs. concentration for epoxy- and CBN-based systems. The dotted red line is the percolation model (equation (2)).

basic mechanism of conduction is related to the dipole motion of polymer chains and spatial charges, which are localized in the phase boundaries between the matrix and electrically conductive clusters with CBN [23].

Figure 2 shows the concentration dependence of direct current electrical conductivity $\left(\sigma_{\mathrm{DC}}(\varphi)\right)$ for the investigated epoxy-CBN systems. It is seen that, with the increase in the filler content in the system, the conductivity increases exponentially, with a percolation transition, which indicates the formation of the conductive network with CBN particles inside the polymer matrix.

The dielectric-conductor transition is partially described by the percolation theory, which is commonly used to establish the relationship between the microstructure of these systems and their physical properties [24, 25]. According to percolation theory, in systems with filler content higher than the percolation threshold, the dependence between the conductivity and the content of the conductive nanofiller is described using Kirkpatrick's model [26]: $\sigma \sim\left(\varphi-\varphi_{c}\right)^{t}$, where $t$ is the critical conductivity exponent, which mainly depends on the topological dimension of the system and does not depend on the structure of the cluster forming particles and from their interaction. However, Kirkpatrick's model allows determining the electrical conductivity only at filler content higher than the percolation threshold. To extend the scope of this model, Efros and Shklovsky proposed using not only Kirkpatrick's equation but also a system of equations as follows [27]:

$$
\sigma_{\mathrm{DC}}=\left\{\begin{array}{ll}
\sigma_{m}\left(\varphi-\varphi_{c}\right)^{t} & \text { Шри } \varphi>\varphi_{c} \\
\sigma_{f}\left(\varphi_{c}-\varphi\right)^{-s} & \text { Шри } \varphi<\varphi_{c}
\end{array},\right.
$$

where $\sigma_{\mathrm{DC}}, \sigma_{m}$, and $\sigma_{f}$ are the electrical conductivity of the composite, matrix, and filler, respectively; $\varphi_{c}$ is the percolation threshold; and $s$ is the critical conductivity exponent that characterizes the number of particles that form a percolation cluster (for a three-dimensional system, the theoretical value is $s \approx 0.73$ [28]). This system of equations is universal and allows describing the electrical conductivity of filled polymer systems in the vicinity of a percolation transition with a high degree of accuracy $[29,30]$.

As a result of modelling the $\left(\sigma_{\mathrm{DC}}(\varphi)\right)$ dependence in the framework of equation (2), the following parameters are obtained: $\varphi_{c}=8 \%$ and $s=0.81$. The obtained value of the critical exponent $s$ is very close to the theoretically calculated one, which indicates the formation of a three-dimensional percolation cluster. The obtained value is much smaller than the value provided by Rebeque $\left(\varphi_{c}=29.3 \%\right)$ for the polyurethane-nanodispersed CB system [23] and Zhang $\left(\varphi_{c}=\right.$ 21\%) for epoxy resin and nanodispersed CB-based systems [13]. However, this value is greater than $\varphi_{c}=3 \%$, obtained by Choi for the nylon-nanodispersed CB system [14], and $\varphi_{c}=2 \%$, obtained by Macutkevic et al. for the epoxy-CB system [31]. This discrepancy in the values of the percolation threshold can be explained by the different dispersion and aggregate capacity of CB particles of different sizes. Thus, according to the authors in [23], who investigated the effect of particle size of $\mathrm{CB}$ on the properties of composites based on polyurethanes, particles of different sizes form different structures in the polymer matrix. Nanoscale particles tend to clump together, forming separate islands separated by large insulation regions. Larger particles of ordinary CB dispersed better, forming a network of conductive clusters in the composite with fewer particles compared to the nanoscale. This behavior is mainly related to the higher density of ordinary $\mathrm{CB}$ particles and their distribution in size, pore structure, and characteristics of the functionalized surface that affect the dispersion in the matrix and the electrical conductivity of the PCM [32].

3.2. Dielectric Properties of Epoxy-Carbon Black Nanopowder Systems. The effect of CBN content in the epoxy composite on the dielectric properties is studied in the next stage of the experiment. Figure 3 shows the frequency dependences of dielectric constant for systems based on the epoxy matrix containing CBN.

Figure 3 presents that the real part of the dielectric constant $(\varepsilon l)$ of the composite materials is almost permanent over a wide frequency range. This effect, probably, is related to the structure of the CBN particles having a carbon core and a shell of different functional groups on the surface, thus creating a minimum gradient of dielectric constant between the matrix and the filler. It is found that, with the introduction and increase in the concentration of the nanoadditive in the epoxy binder, the real part of the dielectric constant also increases.

A graph of the dependence of the real part of the dielectric constant on the CBN content at a frequency of $1 \mathrm{kHz}$ is presented in Figure 4 for more convenient analysis. For better clarity and simplification of modelling, the $\varepsilon^{\prime}(\varphi)$ 


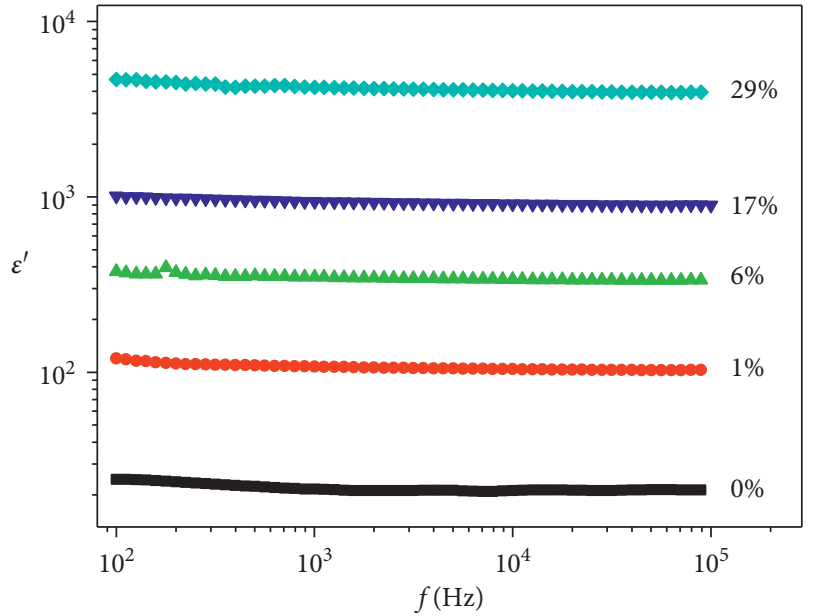

Figure 3: Dielectric constant vs. frequency for systems based on the epoxy matrix containing $\mathrm{CBN}$.

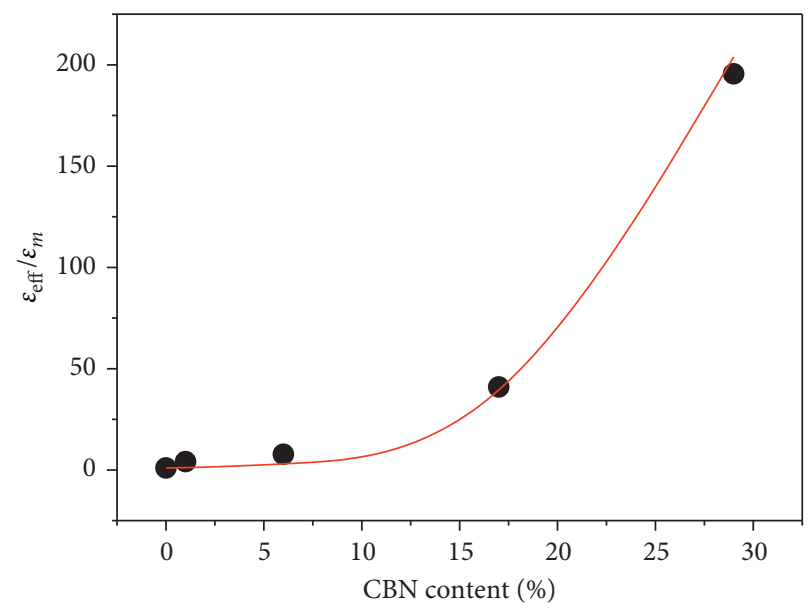

FIGURE 4: Dielectric constant vs. concentration at a frequency of $1 \mathrm{kHz}$ for systems based on the epoxy matrix and CBN. The solid red line is the percolation model (equation (3)).

graph is normalized to the dielectric constant of the polymer matrix. Dependency $\varepsilon l(\varphi)$ graphs at other frequencies have a similar tendency, so they are not given in the paper.

Figure 4 shows a sharp increase in the function with increasing filler content in the system. Such growth is explained by the formation of a branched cluster of $\mathrm{CB}$ nanoparticles in the polymeric matrix. Maxwell-Wagner-Sillars polarization plays an important role in increasing the dielectric constant [21], which is caused by a network of branched electrically conducting clusters of $\mathrm{CB}$ nanoparticles that have not yet formed a percolation cluster. Below the percolation threshold, the distance between the particles of the CBN is not sufficient for hopping or tunnelling. When applying an external electric field on the surfaces of adjacent CBN particles, which are separated by a nonconducting polymeric matrix, the charges opposite in sign are accumulated. In this case, a large number of small capacitors are formed throughout the volume of the nanocomposite, which makes a significant contribution to the dielectric constant of the systems.
According to the scaling approach of critical percolation theory, the concentration dependence of the dielectric constant of a system on the percolation threshold is described by the following equation [33]:

$$
\varepsilon_{\text {eff }}=\varepsilon_{m}\left(\frac{\varphi_{c}}{\varphi_{c}-\varphi}\right)^{q}
$$

where $\varepsilon_{\text {eff }}$ is the dielectric constant of the nanocomposite system; $\varepsilon_{m}$ is the dielectric constant of the matrix; $\varphi_{c}$ is the percolation threshold; and $q$ is the critical dielectric constant exponent, which depends on the experimental results and varies from 0.3 to $1.8[34,35]$. Equation (3) demonstrates that using a matrix with a higher value of $\varepsilon_{m}$, it is possible obtaining a composite material with a high $\varepsilon_{m}$. Another important conclusion from equation (3) is that, in order to obtain a composite with high value of $\varepsilon_{\text {eff }}$, the filler content must be very close to $\varphi_{c}$.

After modelling the data in Figure 4 in the framework of equation (3), it is determined that $\varphi_{c}=26.3 \%$ and $q=0.85$. The value of the critical exponent $q$ is close to the theoretically calculated value $(q=0.73)$ for the three-dimensional system. The percolation threshold values correlate well with those of other polymer-carbon filler systems [23].

It should be noted that when the filler content reached $29 \%$, the obtained material is characterized by a high value of dielectric constant, which in the frequency range from $10^{2}$ to $10^{5} \mathrm{~Hz}$ varies from 4680 to 4000 , (variation is about 15\%). Such values are higher than those obtained for similar systems. For example, Macutkevic et al. for the epoxy-CB system received a maximum value $\varepsilon_{\text {eff }}=100$ [36], Abdelaziz for the system of polycarbonate-nanodispersed $\mathrm{CB}$ received $\varepsilon_{\text {eff }}=250$ [37], and $\mathrm{Xu}$ for epoxy-CB system received $\varepsilon_{\text {eff }}=2300[16]$.

Therefore, the relative frequency invariance of the dielectric constant and its high value make it possible to use the obtained systems as high dielectric constant materials for capacitors and other electrical appliances.

\section{Conclusions}

As a result of this work, the effect of carbon black nanopowder on the electrical properties of epoxy-based polymer composite systems is studied. It is established that the frequency of the electric field and the amount of filler significantly affect the electrical properties of the studied systems. The electrical conductivity of the composite with $29 \%$ CBN shows an increase in only 1-1.5 orders of magnitude, which is explained by the presence of mainly indirect contacts between the filler particles.

The experimental values of AC conductivity for epoxyCBN systems are modelled within the Jonscher equation. The calculated parameters show a decrease in the degree of interaction between charge carriers and the matrix, which is a consequence of the considerable aggregation of CBN particles into denser aggregates whose interaction area with the epoxy polymer is much smaller. It is established that, with increasing filler content in the system, the electrical conductivity increases exponentially, and a percolation 
transition is observed, which indicates the formation of the electrically conductive network inside the polymer matrix. The percolation threshold is $8 \%$. Such a relatively high value can be explained by the high aggregative capacity of the $\mathrm{CB}$ particles.

The features of the dielectric constant of the studied materials are also investigated. It is shown that the dielectric constant of epoxy-CBN systems is almost unchanged in the frequency range of $10^{2}-10^{5} \mathrm{~Hz}$, which is related to the structural features of the filler particles, which ensure the existence of a minimal dielectric gradient between the matrix and the filler. It is found that, at the filler content $29 \%$, the obtained material is characterized by a high dielectric constant, which is 4680 . Therefore, the relative frequency invariance of the dielectric constant and its high value allow the obtained systems to be used as materials with high dielectric constant for electrotechnical devices.

\section{Data Availability}

The data used to support the findings of this study are available from the corresponding author upon request.

\section{Conflicts of Interest}

The authors declare that they have no conflicts of interest regarding the publication of this paper.

\section{References}

[1] S. K. Tiwari, J. Mishra, G. Hatui, and G. C. Nayak, "Conductive polymer composites based on carbon nanomaterials," in Springer Series on Polymer and Composite Materials, pp. 117-142, 2016.

[2] E. A. Lysenkov, O. V. Stryutskiy, Yu. P. Gomza, and V. V. Klepko, "The influence of carbon nanotubes on the sensitivity of humidity sensors based on organic-inorganic polymer materials," Functional Materials, vol. 22, no. 1, pp. 40-46, 2015.

[3] M. H. Naveen, N. G. Gurudatt, and Y.-B. Shim, "Applications of conducting polymer composites to electrochemical sensors: a review," Applied Materials Today, vol. 9, pp. 419-433, 2017.

[4] A. V. Buketov, M. V. Brailo, S. V. Yakushchenko, O. O. Sapronov, and S. O. Smetankin, "The formulation of epoxy-polyester matrix with improved physical and mechanical properties for restoration of means of sea and river transport," Journal of Marine Engineering \& Technology, pp. 1-6, 2018.

[5] A. V. Buketov, O. O. Sapronov, M. V. Brailo, and V. L. Aleksenko, "Influence of the ultrasonic treatment on the mechanical and thermal properties of epoxy nanocomposites," Materials Science, vol. 49, no. 5, pp. 696-702, 2014.

[6] A. V. Buketov, N. A. Dolgov, A. A. Sapronov, V. D. Nigalatii, and N. V. Babich, "Mechanical characteristics of epoxy nanocomposite coatings with ultradisperse diamond particles," Strength of Materials, vol. 49, no. 3, pp. 464-471, 2017.

[7] E. A. Lysenkov, Z. O. Gagolkina, E. V. Lobko, Y. V. Yakovlev, S. D. Nesin, and V. V. Klepko, "Structure-property relationships in polymer nanocomposites based on cross-linked polyurethanes and carbon nanotubes," Functional Materials, vol. 22, no. 3, pp. 342-349, 2015.
[8] A. V. Buketov, A. Sapronov, N. N. Buketova et al., "Impact toughness of nanocomposite materials filled with fullerene C60 particles," Composites: Mechanics, Computations, Applications: An International Journal, vol. 9, no. 2, pp. 141-161, 2018.

[9] K. Kunz, B. Krause, B. Kretzschmar et al., "Direction dependent electrical conductivity of polymer/carbon filler composites," Polymers, vol. 11, no. 4, p. 591, 2019.

[10] Q. Zhang, J. Wang, B.-Y. Zhang, B.-H. Guo, J. Yu, and Z.-X. Guo, "Improved electrical conductivity of polymer/ carbon black composites by simultaneous dispersion and interaction-induced network assembly," Composites Science and Technology, vol. 179, pp. 106-114, 2019.

[11] R. J. Mailabari, O. Mamat, and Z. Baig, "Dispersion of carbon black in epoxy resin and the electrical property of the nanocomposite," Journal of Engineering and Applied Sciences, vol. 11, no. 20, 2016.

[12] E. P. Mamunya, V. V. Davidenko, and E. V. Lebedev, "Effect of polymer-filler interface interactions on percolation conductivity of thermoplastics filled with carbon black," Composite Interfaces, vol. 4, no. 4, pp. 169-176, 1996.

[13] W. Zhang, R. S. Blackburn, and A. A. Dehghani-Sanij, "Carbon black reinforced epoxy resin nanocomposites as bending sensors," Journal of Composite Materials, vol. 43, no. 4, pp. 367-376, 2009.

[14] H.-J. Choi, M. S. Kim, D. Ahn, S. Y. Yeo, and S. Lee, "Electrical percolation threshold of carbon black in a polymer matrix and its application to antistatic fibre," Scientific Reports, vol. 9, no. 1, p. $6338,2019$.

[15] A. V. Buketov, S. A. Smetankin, A. V. Akimov, and A. G. Kulinich, "Epoxy composite modifications influence on the energy activation's of thermal destruction," Functional Materials, vol. 26, no. 2, pp. 403-411, 2019.

[16] J. Xu, M. Wong, and C. P. Wong, "Super high dielectric constant carbon black-filled polymer composites as integral capacitor dielectrics," in Proceedings of the 2004 Proceedings 54th Electronic Components and Technology Conference (IEEE Cat. no 04CH37546), pp. 536-541, IEEE, Las Vegas, NV, USA, June 2004.

[17] Z.-M. Dang, J.-K. Yuan, J.-W. Zha, P.-H. Hu, D.-R. Wang, and Z.-Y. Cheng, "High-permittivity polymer nanocomposites: influence of interface on dielectric properties," Journal of Advanced Dielectrics, vol. 3, no. 3, p. 1330004, 2013.

[18] P. Bertasius, D. Meisak, J. Macutkevic et al., "Fine tuning of electrical transport and dielectric properties of epoxy/carbon nanotubes composites via magnesium oxide additives," Polymers, vol. 11, no. 12, p. 2044, 2019.

[19] A. G. Shaitanov, Y. V. Surovikin, A. D. Morozov, and I. V. Rezanov, "Investigation of conductive nanodisperse carbon by Raman scattering spectroscopy," International Polymer Science and Technology, vol. 40, no. 12, pp. 25-29, 2013.

[20] M. Blanco, G. Kortaberria, A. Jimeno, P. Arruti, A. Tercjak, and I. Mondragon, "Nanocomposites based on polymer matrix and carbon nanotubes as studied by dielectric spectroscopy," in Biopolymers and Nanocomposites as Studied by Dielectric Spectroscopy, vol. 37/661, pp. 1-35, , no. 2, Transworld Research Network, ISBN: 978-81-7895-399-1, Transworld Research Network, 2009, ISBN: 978-81-7895-399-1.

[21] F. Kremer and A. Schönhals, Broadband Dielectric Spectroscopy, Springer-Verlag, Berlin, Germany, 2003.

[22] S. Sinha, S. K. Chatterjee, J. Ghosh, and A. K. Meikap, "Analysis of the dielectric relaxation and ac conductivity behavior of polyvinyl alcohol-cadmium selenide 
nanocomposite films," Polymer Composites, vol. 38, no. 2, pp. 287-298, 2017.

[23] P. Vinı'cius Rebeque, M. Jones Silva, C. Rafael Cena, H. Naoyuki Nagashima, J. Antonio Malmonge, and D. H. Fujii Kanda, "Analysis of the electrical conduction in percolative nanocomposites based on castor-oil polyurethane with carbon black and activated carbon nanopowder," Polymer Composites, vol. 40, no. 1, pp. 7-15, 2019.

[24] S. D. McCullen, D. R. Stevens, W. A. Roberts, S. S. Ojha, L. I. Clarke, and R. E. Gorga, "Morphological, electrical, and mechanical characterization of electrospun nanofiber mats containing multiwalled carbon nanotubes," Macromolecules, vol. 40, no. 4, pp. 997-1003, 2007.

[25] V. V. Klepko and E. A. Lysenkov, "Features of percolation transition in systems on the basis of oligoglycols and carbon nanotubes," Ukrainian Journal of Physics, vol. 60, no. 9, pp. 944-949, 2015.

[26] S. Kirkpatrick, "Classical transport in disordered media: scaling and effective-medium theories," Physical Review Letters, vol. 27, no. 25, pp. 1722-1725, 1971.

[27] A. L. Efros and B. I. Shklovskii, "Critical behaviour of conductivity and dielectric constant near the metal-non-metal transition threshold," Physica Status Solidi (B), vol. 76, no. 2, pp. $475-485,1976$.

[28] J. P. Clerc, G. Giraud, J. M. Laugier, and J. M. Luck, "The electrical conductivity of binary disordered systems, percolation clusters, fractals and related models," Advances in Physics, vol. 39, no. 3, pp. 191-309, 1990.

[29] E. A. Lysenkov and V. V. Klepko, "Analysis of percolation behavior of electrical conductivity of the systems based on polyethers and carbon nanotubes," Journal of Nano-and Electronic Physics, vol. 8, no. 1, p. 01017, 2016.

[30] E. A. Lysenkov and V. V. Klepko, "Features of percolation transition in nanocomposites based on polyethers and carbon nanotubes," Journal of Physical Studies, vol. 20, no. 3, p. 3702, 2016.

[31] J. Macutkevic, P. Kuzhir, A. Paddubskaya et al., "Epoxy resin/ carbon black composites below the percolation threshold," Journal of Nanoscience and Nanotechnology, vol. 13, no. 8, pp. 5434-5439, 2013.

[32] R. A. Antunes, M. C. L. De Oliveira, G. Ett, and V. Ett, "Carbon materials in composite bipolar plates for polymer electrolyte membrane fuel cells: a review of the main challenges to improve electrical performance," Journal of Power Sources, vol. 196, no. 6, pp. 2945-2961, 2011.

[33] L. Zhang, W. Wang, X. Wang, P. Bass, and Z.-Y. Cheng, "Metal-polymer nanocomposites with high percolation threshold and high dielectric constant," Applied Physics Letters, vol. 103, no. 23, Article ID 232903, 2013.

[34] L. Wang and Z.-M. Dang, "Carbon nanotube composites with high dielectric constant at low percolation threshold," Applied Physics Letters, vol. 87, no. 4, Article ID 042903, 2005.

[35] J. Macutkevic, D. Seliuta, G. Valušis et al., "High dielectric permittivity of percolative composites based on onion-like carbon," Applied Physics Letters, vol. 95, no. 11, Article ID 112901, 2009.

[36] J. Macutkevic, P. Kuzhir, A. Paddubskaya et al., "Electrical transport in carbon black-epoxy resin composites at different temperatures," Journal of Applied Physics, vol. 114, no. 3, Article ID 033707, 2013.

[37] M. Abdelaziz, "The effects of carbon nanoparticles on thermal and dielectric properties of bisphenol A polycarbonate," Journal of Thermoplastic Composite Materials, vol. 28, no. 7, pp. 1026-1046, 2015. 